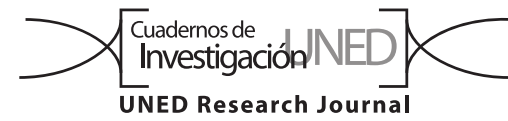

\title{
Permanencia de la cobertura natural en dos propuestas de conectividad entre áreas silvestres protegidas de Costa Rica
}

\author{
Michael Steven Arroyo Zeledón' \& Carlos Morera Beita ${ }^{1}$ \\ 1. Universidad Nacional, Heredia, Costa Rica; michaelsteven.arroyo@gmail.com,cmorera@una.com \\ Recibido 01-XI-2018 • Corregido 12-XII-2018 • Aceptado 30-I-2019
}

\begin{abstract}
Natural cover permanence in two connectivity proposals between protected wildlife areas of Costa Rica". Introduction: Riparian zones are an alternative for connectivity in landscapes where natural cover has been fragmented. Objective: To analyze structural connectivity between two protected wildlife areas of Costa Rica, through riparian zones, and an officially established route. Methods: The two protected wildlife areas were Lomas Barbudal Biological Reserve and Miravalles Protective Zone, Guanacaste province. Using supervised classification of Landsat images from years 1997 and 2017, land cover was obtained for riparian zones and the official route. Based on this information, forest permanence was analyzed and several fragmentation indicators calculated for the two connectivity proposals. Results: Forest was more extensive and permanent in the riparian zones; nevertheless, both connectivity proposals had interruptions in connectivity and isolated forest sectors. In the official route, interruption was caused mainly by urban areas; riparian zones were interrupted by agriculture. Conclusion: Riparian zones would also provide good structural connectivity, but they need to recover such connectivity too.
\end{abstract}

Key words: structural connectivity, dispersal routes, protected wildlife areas, natural cover permanence, riparian zones.
RESUMEN: Introducción: Las zonas ribereñas constituyen una alternativa para lograr conectividad en paisajes donde la cobertura natural ha sido fragmentada. Objetivo: Analizamos la conectividad estructural entre dos áreas silvestres protegidas de Costa Rica mediante zonas ribereñas y una ruta oficialmente establecida. Métodos: Las áreas silvestres protegidas fueron la Reserva Biológica Lomas Barbudal y la Zona Protectora Mirallaves, ubicadas hacia el noroeste del país, en la provincia Guanacaste. A partir de la clasificación supervisada de dos imágenes Landsat de los años 1997 y 2017, obtuvimos la cobertura de la tierra en las zonas ribereñas y la ruta oficial. Con base en esta información analizamos la permanencia del bosque en las dos propuestas de conectividad y calculamos varios indicadores de fragmentación. Resultados: El bosque fue más extenso y permanente en las zonas ribereñas que en la ruta oficial; no obstante, en ambas propuestas de conectividad se encontraron sectores con concentración de cobertura boscosa distantes entre sí y una interrupción de tal conectividad. En el caso de la ruta oficial, la interrupción se debió principalmente a áreas urbanas mientras que en las zonas ribereñas al desarrollo agrícola. Conclusión: A partir de los hallazgos del presente estudio, resulta razonable proponer las zonas ribereñas como rutas de conectividad estructural entre las áreas silvestres protegidas involucradas; sin embargo, tanto en estas zonas como en la ruta oficial es necesario recuperar dicha conectividad.

Palabras clave: conectividad estructural, rutas de conectividad, áreas silvestres protegidas, permanencia de cobertura natural, zonas ribereñas.
Los bosques tropicales proporcionan variedad de bienes y servicios a las comunidades humanas debido a la diversidad biológica que albergan (Balvanera, 2012). Por consiguiente, extensiones considerables de bosques se conservan en áreas protegidas (Joppa, Loarie, \& Pimm, 2008; Portillo-Quintero \& Sánchez-Azofeifa, 2010). Sin embargo, el aislamiento de poblaciones de flora y fauna en fragmentos de bosque dentro de áreas protegidas amenaza la supervivencia de dichas poblaciones (DeFries, Hansen, Newton, \& Hansen, 2005; Laurance et al., 2012) y Costa Rica no ha estado exenta de este problema (Sanchez-Azofeifa, Rivard, Calvo, \& Moorthy, 2002; Sánchez-Azofeifa, Daily, Pfaff, \& Busch, 2003).

En el 2007 se identificaron, de forma oficial, las rutas de conectividad entre las áreas silvestres protegidas ubicadas en la plataforma continental del territorio costarricense (Sistema Nacional de Áreas de Conservación [SINAC], 2007). Estas rutas constituyen elementos importantes para la conservación de biodiversidad en el país (Arias, Chacón, Herrera, Induni, Acevedo, Coto, \& Barborak, 2008) pero presentan dos inconvenientes principales. En primera instancia, hay trayectorias sin 
cobertura natural que se localizan parcial o totalmente en terrenos de propiedad privada (Arauz-Beita \& AriasNavarro, 2016; Quesada, 2016) donde el Estado tiene poca injerencia para facilitar su restauración.

El segundo inconveniente de las rutas de conectividad oficiales entre áreas silvestres protegidas de Costa Rica es que muchas están interrumpidas por la red vial (Artavia et al., 2015). Desde hace casi dos décadas se tiene registro de que en el territorio costarricense las carretas ocasionan mortalidad de fauna (Monge-Nájera, 1996; Carvajal \& Díaz, 2016) y pocas de estas tienen estructuras para facilitar el paso de animales (Artavia et al., 2015). La situación constituye una limitante para la cual las soluciones aún no están totalmente definidas (Torres, 2011; Araya-Gamboa \& Salom-Pérez, 2015).

Durante la identificación de rutas de conectividad entre áreas silvestres protegidas del territorio costarricense se reconoció la importancia de las zonas ribereñas (SINAC, 2007). Sin embargo, por sí mismas estas zonas no constituyen vías de comunicación oficiales, a pesar de la conocida importancia de los elementos lineales del paisaje para la conectividad (Treviño, Cavazos, \& Aguirre, 2001; Sastre, De Lucio, \& Martínez, 2002). Además, la Ley Forestal de Costa Rica (número 7575), en su artículo 33, define áreas de protección para la red fluvial y esto contribuye a su conservación. En otros países de mayor diversidad biológica como Brasil se aplica una legislación similar y constituye un respaldo para estrategias dirigidas a mejorar la conectividad entre áreas protegidas mediante la vegetación boscosa en riberas de ríos y quebradas (Lees \& Peres, 2007).

Un alto número de especies vegetales tiene asociación con zonas ribereñas, lo que unido al microclima húmedo convierte a estas últimas en hábitats favorables para la fauna (Granados-Sánchez, Hernández-García, \& LópezRíos, 2006; Pérez et al., 2006). Esta fauna incluye especies para las que ha disminuido su hábitat natural en Costa Rica, como es el caso del chirrascuá, Dendrortyx leucophrys (Sandoval, Sánchez, \& Carman, 2013). Además, se tiene evidencia de que en el territorio costarricense, incluso en áreas urbanas, el bosque ribereño actúa como fuente de propágulos para la regeneración vegetal en áreas desprovistas de vegetación (Di Stéfano, Nielsen, Hoomans, \& Fournier, 1996).

En Costa Rica, luego de evaluar la efectividad de manejo de los corredores biológicos, Canet-Desanti, Herrera y Finegan (2012) concluyeron que era necesario continuar investigando sobre los componentes de conservación como el desarrollo de conectividad entre fragmentos de bosque. Existe la amenaza de que los fragmentos más extensos queden aislados dentro de áreas silvestres protegidas (Broadbent et al., 2012). Por este motivo, entre los principios orientadores de las políticas nacionales para dichas áreas se encuentra el desarrollo de conectividad, como medio para garantizar la viabilidad de la biodiversidad a largo plazo (SINAC, 2010), y es sugerido por la teoría de biogeografía de islas (MacArthur \& Wilson, 1967).

A partir de lo anterior, el objetivo de esta investigación fue analizar, por medio de la permanencia de la cobertura natural, la conectividad estructural entre dos áreas silvestres protegidas de Costa Rica mediante zonas ribereñas y una ruta oficialmente establecida.

\section{MATERIALES Y MÉTODOS}

Este estudio involucró las siguientes áreas silvestres protegidas de la cuenca del río Bebedero: la Reserva Biológica Lomas Barbudal y la Zona Protectora Miravalles. Estas áreas se ubican al noroeste de Costa Rica, entre las coordenadas geográficas $10^{\circ} 25^{\prime} 32^{\prime \prime}-10^{\circ} 47^{\prime} 58^{\prime \prime} \mathrm{N}$ \& $85^{\circ} 4^{\prime} 37^{\prime \prime}-85^{\circ} 22^{\prime} 55^{\prime \prime}$ W. Desde el año 2007 existe entre ambas áreas protegidas la ruta de conectividad oficial número 115 identificada por el Proyecto GRUAS II (SINAC, 2007). Sin embargo, otra alternativa de conectividad corresponde a las zonas ribereñas de los ríos Piedras Blancas y Tenorio, afluentes del río Bebedero (Fig. 1).

Para realizar un análisis comparativo de la permanencia de la cobertura natural en las dos propuestas de conectividad estructural, se estableció un área de $1,5 \mathrm{~km}$ de ancho a cada lado de ambas y paralela a la trayectoria de estas. El ancho del área corresponde a tres veces el sugerido como óptimo para las rutas de conectividad en Costa Rica (Gómez, 2014).

La permanencia de la vegetación natural en las dos propuestas de conectividad estructural se analizó a partir de la cobertura de la tierra alrededor de ambas para los años 1997 y el 2017. Dicha cobertura de la tierra se obtuvo de la clasificación supervisada de dos imágenes Landsat para esos años utilizando el programa de cómputo ERDAS Imagine. Estos archivos tienen un tamaño de píxel de $30 \mathrm{~m}$ y fueron captadas entre los meses enero y febrero de los años señalados. La poca nubosidad y calidad de las imágenes hizo innecesaria la aplicación de algún tipo de corrección. El área mínima de mapeo fue de $2 * 2$ píxeles $(0,36 \mathrm{ha})$, como ha sido sugerido para estas imágenes (Grinand et al., 2013).

Para mejorar la clasificación supervisada de la imagen de 1997 se usaron fotografías aéreas (1997-1998) no georreferenciadas del proyecto Terra (Centro Nacional de Información Geoambiental, 2018) mediante la 


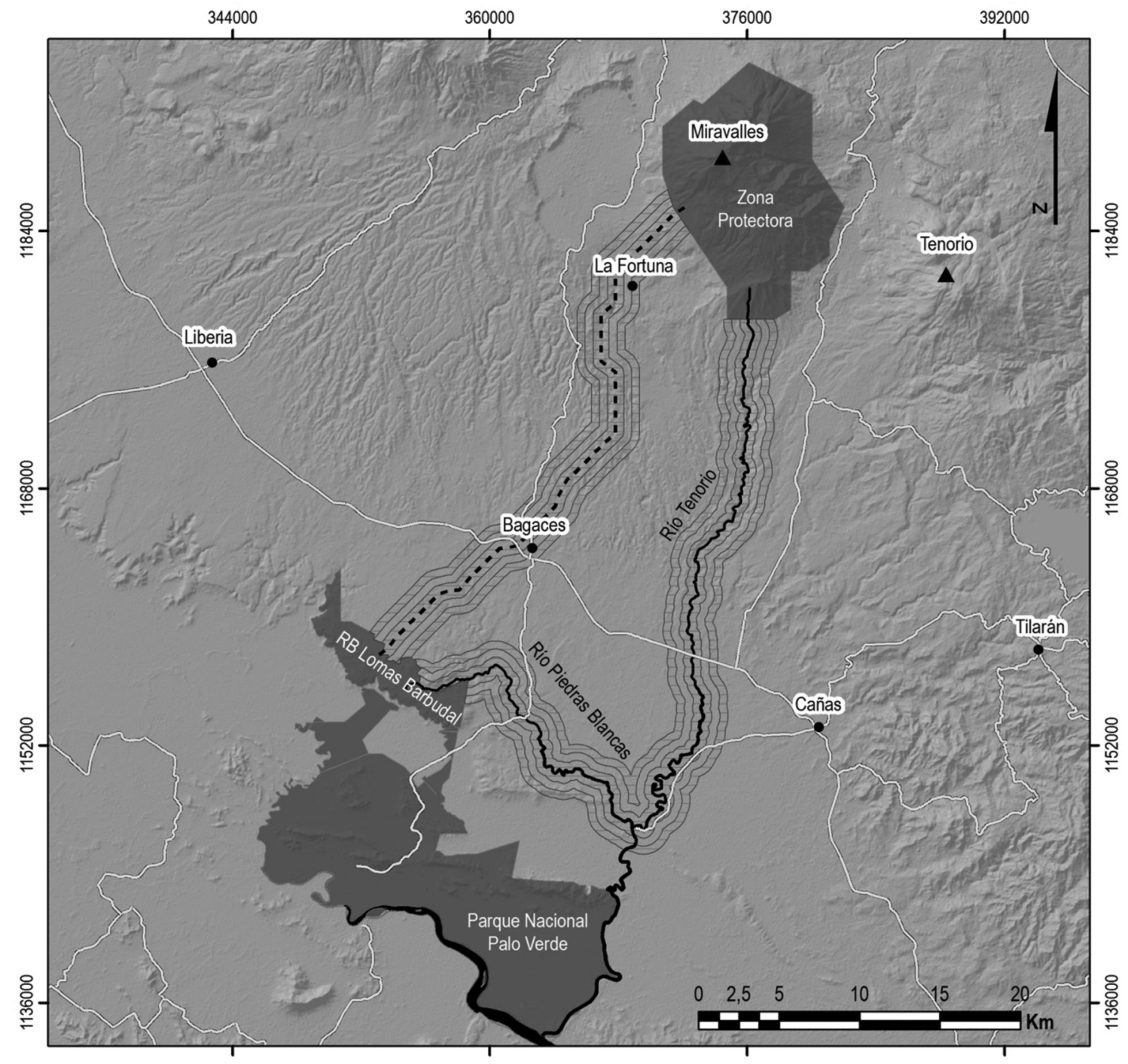

\section{Simbología}

- Poblado

- Volcán Carretera

- - - - Ruta de conectividad oficial - Ruta de conectividad alternativa Bandas de análisis Área Silvestre Protegida

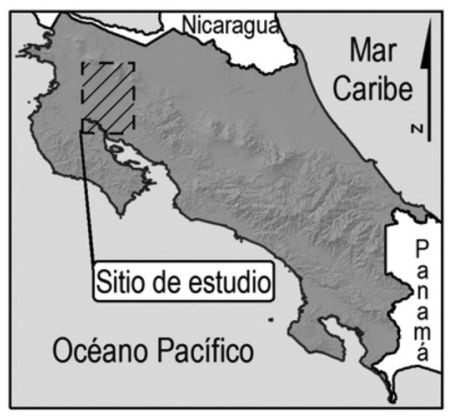

Fig. 1. Ubicación de las propuestas de conectividad estructural analizadas. 


\begin{tabular}{ll}
\multicolumn{1}{c}{ Categoría } & \multicolumn{1}{c}{ Descripción } \\
Bosque & Bosque permanente y deciduo, desde etapas preliminares de sucesión hasta estadios avanzados. \\
Cuerpo de agua & Cuerpos de agua estacionarios o en movimiento, con poca o mucha intervención humana en su génesis. \\
Plantación forestal & Área dedicada al cultivo de árboles maderables. Para el área de estudio domina la especie Tectona grandis (teca). \\
Pasto & Áreas con gramíneas que pueden incluir árboles dispersos y utilizarse para la alimentación de ganado bovino. \\
Cultivo & Áreas dedicadas al cultivo agrícola permanente o estacional. \\
Infraestructura & Áreas ocupadas por paneles solares para la generación de energía eléctrica. \\
Urbano & Territorio ocupado por viviendas y comercio. \\
Terreno descubierto & Área desprovista de cobertura vegetal, con suelo en roca, lastre o concreto. \\
\hline
\end{tabular}

identificación en ellas de puntos de referencia. La clasificación supervisada de la imagen del 2017 se apoyó en imágenes Bing de alta resolución y disponibles mediante la herramienta ArcBruTile del programa de cómputo ArGIS. Adicionalmente, se verificó en campo la cobertura actual de la tierra. Las categorías de cobertura identificadas fueron: bosque, cuerpo de agua, plantación forestal, pasto, cultivo, infraestructura, urbano y terreno descubierto (Cuadro 1).

La confiabilidad de la clasificación supervisada de la imagen del 2017 se evaluó mediante una matriz de confusión. Esta valora los registros de cobertura tomados en campo con los resultados de la clasificación (Cuadro 2). La confiabilidad se obtiene como el porcentaje de los aciertos (Fernández, Recio, \& Ruiz, 2003; François, Reyes, \& Pérez, 2003; Chuvieco, 2010) y para este estudio fue de $87,8 \%$, la cual es considerada aceptable y alta (François et al., 2003; Valdez-Lazalde, Aguirre-Salado, \& ÁngelesPérez, 2011).

Con el propósito de comparar la permanencia de la cobertura natural a mayor y menor cercanía de las dos propuestas de conectividad, el área de análisis a ambos lados de éstas (1,5km de ancho) fue dividida en tres bandas paralelas de $500 \mathrm{~m}$. De esta forma se establecieron las siguientes bandas de análisis a ambos lados de las dos propuestas: banda $1(0-500 \mathrm{~m})$, banda $2(500-1000 \mathrm{~m})$ y banda 3 (1 000-1 500m).

La permanencia de la cobertura natural en las dos propuestas de conectividad fue determinada con el Modelador de Cambio en el Terreno (mejor conocido en inglés como Land Change Modeler) del programa de cómputo IDRISI. Los cálculos de área de las distintas categorías de cobertura de la tierra se realizaron con el programa ArcGIS.

En el área de análisis establecida para las dos propuestas de conectividad $(1,5 \mathrm{Km}$ de ancho a ambos lados de estas) se calcularon los siguientes indicadores de fragmentación del bosque: número de fragmentos, tamaño medio de estos con su desviación estándar y distancia mínima promedio entre ellos (Reyes, Morera, \& Reyes, 2017). Estos indicadores se obtuvieron con ayuda de los programas de cómputo ArcGIS 10.1, Fragstats 4.2 y

\section{CUADRO 2}

Matriz de confusión para la clasificación supervisada de la imagen del 2017

\begin{tabular}{|c|c|c|c|c|c|c|c|c|c|}
\hline Cobertura & B & $C A$ & PF & $\mathrm{P}$ & $C$ & I & U & TD & Total \\
\hline B & 20 & & 1 & & & & & & 21 \\
\hline$C A$ & & 1 & & & & & & & 1 \\
\hline PF & & & 4 & 1 & & & & & 5 \\
\hline$P$ & & & & 27 & 4 & & & & 31 \\
\hline C & & & & 1 & 13 & & & 1 & 15 \\
\hline I & & & & & & 1 & & & 1 \\
\hline U & & & & & & & 2 & & 2 \\
\hline TD & & & & 2 & & & & 4 & 6 \\
\hline Confiabilidad, acierto total: $87,8 \%$ & & & & & & & & & 82 \\
\hline
\end{tabular}

Nota. Coberturas de la tierra, B: bosque, CA: cuerpo de agua, PF: plantación forestal, P: pasto, C: cultivo, I: Infraestructura, U: urbano, TD: terreno descubierto. Los números en la diagonal corresponden a aciertos según registros tomados en el sitio de estudio en cuanto a cobertura de la tierra. 
SPSS Statistics 21.0. Además, se calculó el índice de fragmentación (IF) propuesto por Vargas (2008) y ajustado por Morera y Sandoval (2018). Mayores valores de este índice señalarán un paisaje más fragmentado, sea por un aumento en el número de fragmentos o por la mayor distancia entre estos.

$$
\mathrm{IF}=\frac{\mathrm{SPTA} * 100}{\mathrm{Sm}(\Sigma \mathrm{Nm} / \mathrm{Dm})}
$$

IF: Índice de fragmentación.

SPTA: Superficie total del área.

Sm: Superficie de los fragmentos.

$\mathrm{Nm}$ : Número total de fragmentos.

Dm: Distancia entre fragmentos medida desde el centro de estos.

Para los fragmentos de bosque que se extendían más allá del área de análisis de cada propuesta de conectividad se tuvo el siguiente criterio de decisión: si más del $50 \%$ de la extensión del fragmento se encontraba en dicha área fue incluido en el análisis y sin que esta última lo dividiera. Este criterio tuvo como propósito enfocarse en la extensión real de los fragmentos.

Ética, conflicto de intereses y declaración de financiamiento: los autores declaran haber cumplido con todos los requisitos éticos y legales pertinentes, tanto durante el estudio como en el manuscrito; que no hay conflictos de interés de ningún tipo, y que todas las fuentes financieras se detallan plena y claramente en la sección de agradecimientos. Asimismo, están de acuerdo con la versión editada final del documento. El respectivo documento legal firmado se encuentra en los archivos de la revista.

\section{RESULTADOS}

En 1997 y el 2017, el pasto fue la cobertura de mayor extensión en la ruta de conectividad oficial mientras que el bosque en la ruta alterna (Fig. 2). En 1997, se identificaba en la ruta oficial una matriz dominada por pasto con cobertura boscosa dispersa mientras que en la ruta alterna un escenario similar pero con un área de cultivos agrícolas que separaba sectores con concentración de bosque hacia el norte y el sur. Para el 2017 son más evidentes los sectores que concentran cobertura boscosa, particularmente hacia el noreste de las dos propuestas de conectividad (Apéndice Fig. A).

Aunque la interrupción de la conectividad estructural en la ruta oficial no es tan extensa como en la alterna, también está presente. La ruta oficial es interrumpida tanto por pasto como por las áreas urbanas de Bagaces y La Fortuna (Apéndice Fig. A).

Tanto en 1997 como en el 2017 hubo una disminución del área de bosque desde la banda más cercana hacia la más distante de cada propuesta de conectividad. La excepción ocurrió de la segunda a la tercera banda en la ruta oficial. Sin embargo, en esa ruta sí se presentó una disminución del área boscosa de la primera a la segunda banda en ambos años. La reducción en la extensión del bosque de la primera a la segunda banda fue más marcada en la ruta alterna para los dos años (Fig. 3).

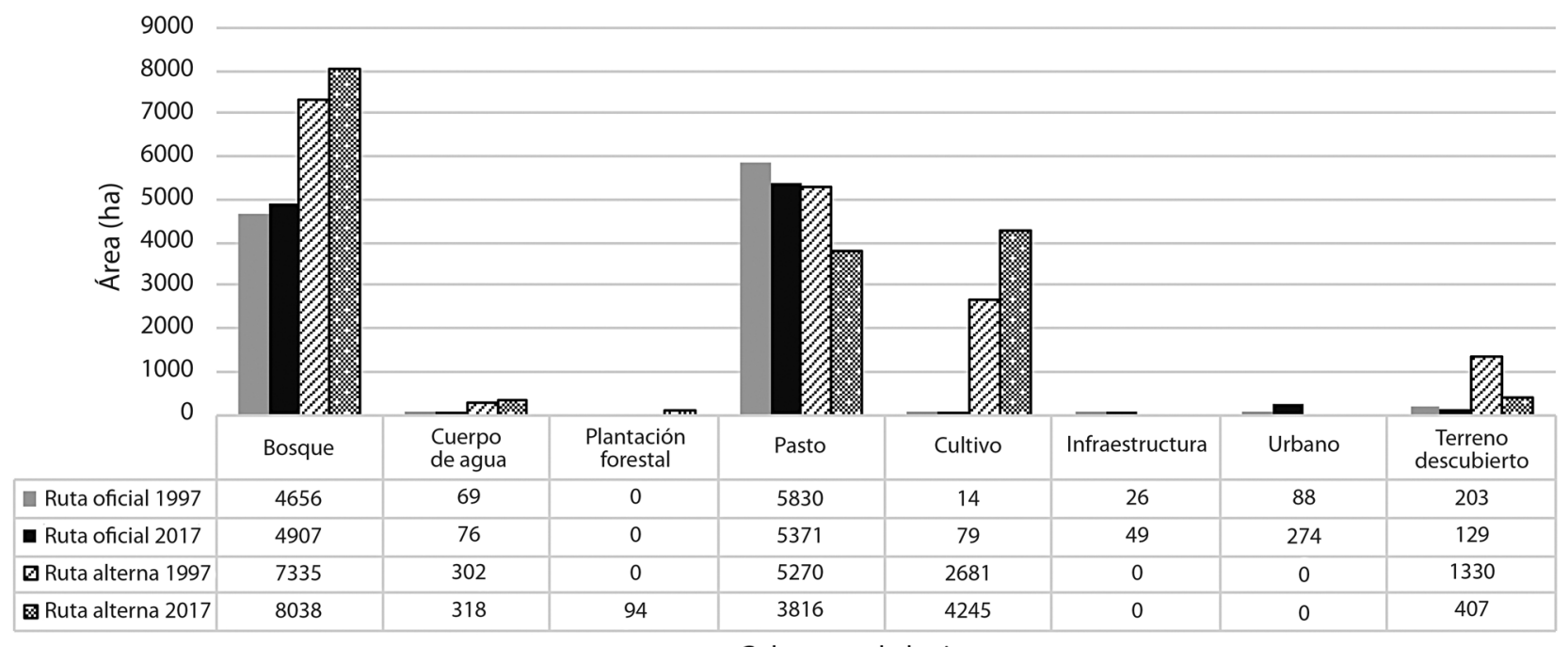

Cobertura de la tierra

Fig. 2. Área ocupada por las distintas coberturas de la tierra. 


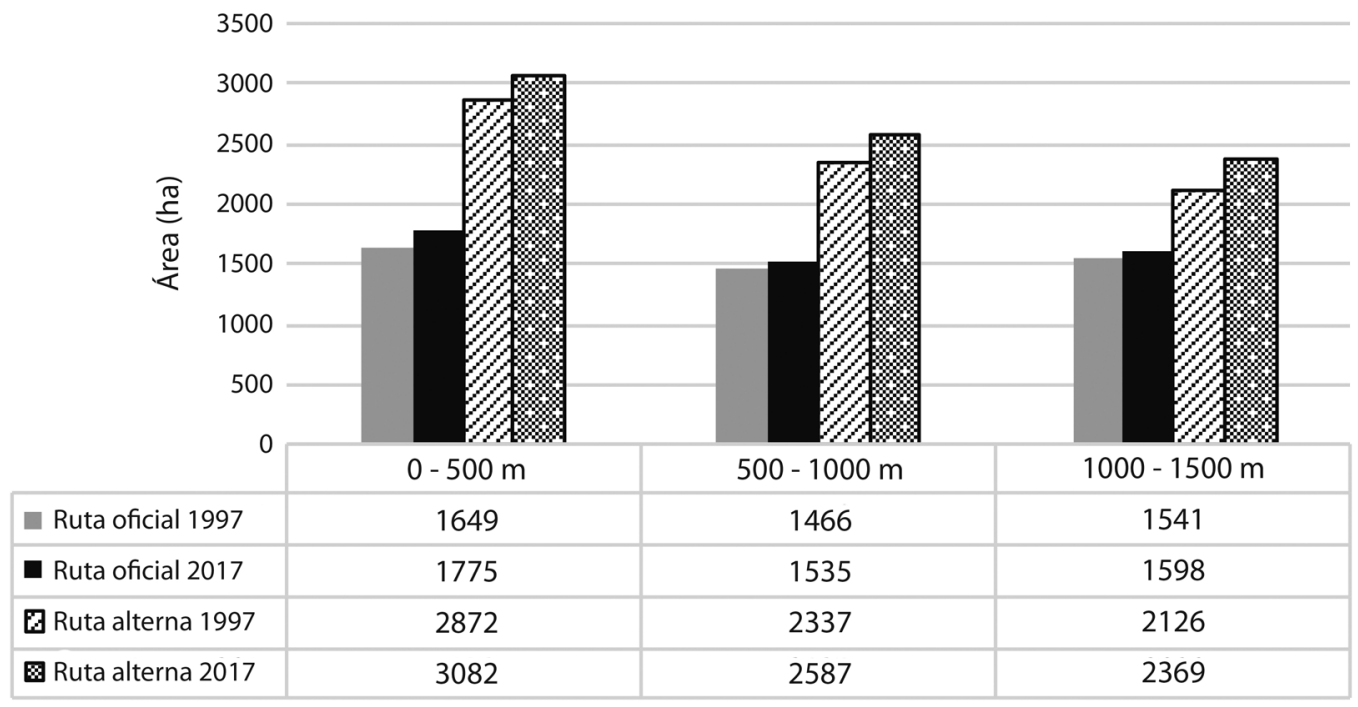

Bandas de análisis

Fig. 3. Área de bosque en las tres bandas paralelas a las dos propuestas de conectividad.

Las dos propuestas de conectividad presentaron un aumento en el área ocupada por bosque entre 1997 y 2017 (Fig. 2). Para la ruta oficial, el incremento fue de $252 \mathrm{ha}(5 \%)$ aproximadamente mientras que para la ruta alterna 703ha (9\%). Este aumento en el área boscosa también se refleja en las bandas paralelas a las propuestas (Fig. 3). El incremento del área con bosque en ambas propuestas ocurrió a expensas de terreno cubierto por pasto (Fig. 4). También se encontró que alrededor de 84 ha de cobertura boscosa se perdieron en la ruta oficial como consecuencia del crecimiento urbano en tanto que en la ruta alterna aproximadamente 261 ha de bosque a expensas de cultivo agrícola (Fig. 4).

En cualquiera de las bandas consideradas, la permanencia de la cobertura boscosa fue mayor en la ruta alterna que en la oficial. El incremento de área ocupada por bosque fue también superior en cualquiera de las bandas de la ruta alterna (Cuadro 3). En la ruta oficial, el aumento de área boscosa estuvo concentrado hacia el norte mientas que las pérdidas son más notorias hacia el sur. Las pérdidas en la ruta alterna son igualmente más evidentes hacia el sur aunque también existió concentración de ganancias en ese sector (Apéndice Fig. B).

Los resultados obtenidos con los indicadores de fragmentación también reflejan un aumento en la concentración del bosque en sectores de ambas propuestas de conectividad. En las dos ocurrió una reducción en el número de fragmentos de bosque entre 1997 y 2017. Además, el tamaño medio de estos se amplió, al igual que la distancia mínima promedio entre ellos. Consecuente con esto último, de un año a otro el índice de fragmentación se incrementó en ambas propuestas de conectividad (Cuadro 4).

\section{CUADRO 3}

Área de bosque perdida, ganada y permanente en las tres bandas paralelas a las dos propuestas de conectividad

\begin{tabular}{lcccccc}
$\begin{array}{c}\text { Bandas de } \\
\text { análisis }\end{array}$ & \multicolumn{3}{c}{ Área de bosque (ha) } & \multicolumn{2}{c}{ Ruta alterna } \\
Banda 1 & Reducción & $\begin{array}{c}\text { Ruta oficial } \\
\text { Incremento }\end{array}$ & Permanencia & Reducción & Incremento & Permanencia \\
Banda 2 & 457 & 583 & $(72 \%)^{*}$ & 429 & 639 & $(85 \%)$ \\
Banda 3 & 449 & 518 & $(69 \%)$ & 381 & 630 & $(84 \%)$ \\
\hline
\end{tabular}

Nota. * Porcentajes con respecto al área de bosque en 1997. 
A

Pérdida de bosque Aumento de bosque

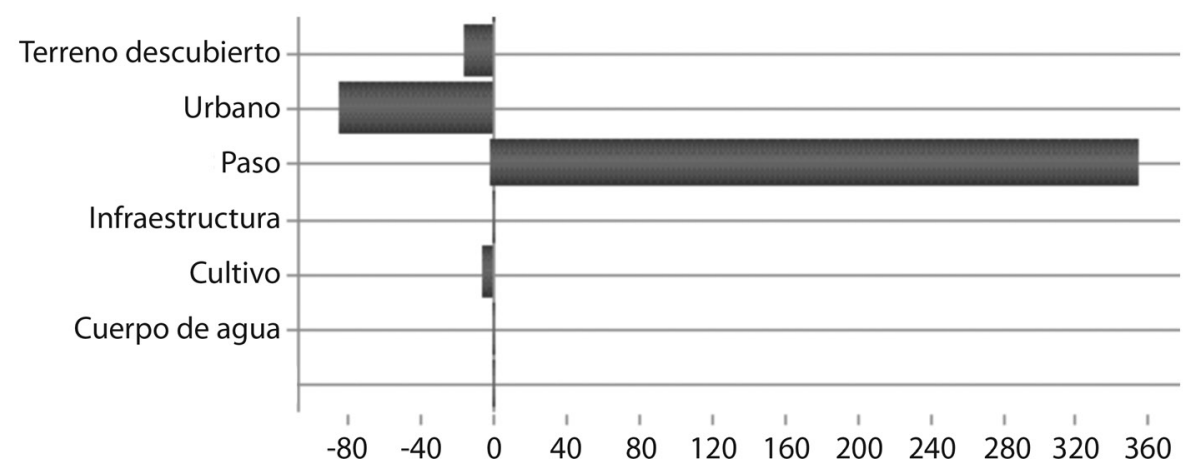

B

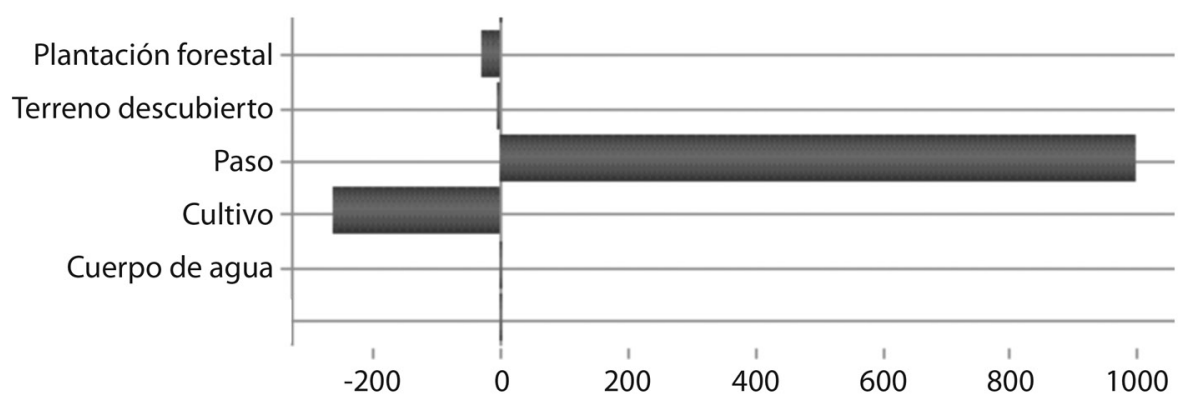

Fig. 4. Pérdidas y aumentos (en ha) de área boscosa explicados por distintas coberturas de la tierra en las dos propuestas de conectividad. a) ruta oficial, b) ruta alterna.

CUADRO 4

Tamaño medio de fragmentos de bosque, distancia mínima promedio entre estos e índice de fragmentación en las dos propuestas de conectividad

\begin{tabular}{|c|c|c|c|c|}
\hline \multirow{2}{*}{ Indicador } & \multicolumn{2}{|c|}{ Ruta oficial } & \multicolumn{2}{|c|}{ Ruta alterna } \\
\hline & 1997 & 2017 & 1997 & 2017 \\
\hline Número de fragmentos & 365 & 296 & 318 & 260 \\
\hline Tamaño medio fragmentos (ha) & $11( \pm 74)$ & $14( \pm 68)$ & $20( \pm 179)$ & $26( \pm 164)$ \\
\hline DMP* entre fragmentos $(\mathrm{m})$ & $70( \pm 45)$ & $83( \pm 56)$ & $85( \pm 120)$ & $109( \pm 158)$ \\
\hline Índice de fragmentación & 51 & 76 & 72 & 106 \\
\hline
\end{tabular}

Nota. \pm Desviación estándar; * DMP: Distancia mínima promedio.

\section{DISCUSIÓN}

La mayor extensión y permanencia del bosque en la ruta alterna destaca el valor de las zonas ribereñas como rutas de conectividad entre áreas silvestres protegidas. Es bien conocido que el bosque como cobertura natural facilita la conectividad (Martensen, Pimentel, \& Metzger, 2008; Haddad et al., 2015). También debe destacarse que la persistencia de la cobertura natural en rutas de conectividad es requisito para mantener en el largo plazo la comunicación entre áreas protegidas, de manera que se favorezca la conservación de diversidad biológica (Ramos \& Finegan, 2004; Piquer-Rodríguez, Kuemmerle, Alcaraz-Segura, Zurita-Milla, \& Cabello, 2012). A esto se suma que cuando las zonas ribereñas son intersecadas por la red vial, los puentes involucrados pueden funcionar como paso subterráneo para la vida silvestre (Jackson \& Griffin, 2000). Además, estas zonas cuentan en Costa Rica con cierto grado de protección otorgado por la Ley Forestal en su artículo 33. 
A partir de los hallazgos del presente estudio, es razonable proponer que las zonas ribereñas constituyan también rutas oficiales de conectividad y de esa manera aumentar la probabilidad de que las áreas silvestres protegidas involucradas se mantengan comunicadas. Otros autores ya han destacado el valor de estas zonas como rutas de conectividad (Gurrutxaga \& Lozano, 2007; García \& Abad, 2014). Sin embargo, los resultados obtenidos también develan la necesidad de recuperar la conectividad estructural en determinados sectores tanto de la ruta oficial como de las zonas ribereñas.

La interrupción de la conectividad estructural que ocurre en la ruta oficial por áreas urbanas está reconocida en la literatura como una de las limitantes principales para dicha conectividad (Matteucci, 2010). En algunos trabajos estas áreas han llegado a ser denominadas barreras para la conectividad (McRae, Hall, Beier, \& Theobald, 2012). Hasta el momento, el desarrollo de conectividad estructural en sitios urbanos es un campo de investigación en etapas iniciales (LaPoint, Balkenhol, Hale, Sadler, \& van der Ree, 2015), por lo que en el corto plazo resulta válido proponer que en el área de estudio se modifique la trayectoria de la ruta oficial para que se distancie lo más posible de los poblados Bagaces y La Fortuna.

En el caso de la ruta alterna, también es conocido que la agricultura interrumpe la conectividad estructural (Matteucci, 2010) pero en comparación con la urbanización se dispone de más soluciones para recuperar dicha conectividad en terrenos agrícolas. La instauración de cercas vivas o la incorporación de cobertura arbórea son algunas acciones que han funcionado para restablecer la conectividad en áreas dedicadas a agricultura (De la Ossa-Lacayo, 2013; Sanfiorenzo, DeClerck, Benjamin, \& Velásquez, 2011). En el sector agrícola de la ruta alterna, las acciones pueden iniciarse en el área de protección de ríos que dicta la Ley Forestal de Costa Rica, de manera que se cuente con respaldo normativo.

En el área de estudio resulta positivo para la conectividad el incremento de cobertura boscosa en terreno previamente ocupado por pasto y esto ha sido registrado en toda la provincia Guanacaste (Tapia, 2016). Sin embargo, el hallazgo de este estudio en cuanto a la concentración de la cobertura boscosa en sectores distantes entre sí respalda la siguiente conclusión para los esfuerzos de conservación de biodiversidad: la recuperación del bosque debe estar estratégicamente dirigida para que propicie la conectividad entre áreas silvestres protegidas, disminuyendo la distancia entre fragmentos boscosos. Autores como Volk, Gattringer, Otte y HarvolkSchöning (2018) han planteado que la mejora en la conectividad funcione como un indicador del éxito de los programas de restauración de cobertura vegetal en determinado territorio.

La interrupción de la conectividad estructural en las dos propuestas analizadas amenaza la conservación de biodiversidad. Uno de los factores que puede incidir en la fragmentación encontrada es que ni siquiera las rutas oficiales de conectividad están protegidas como tales en la normativa costarricense. Sin embargo, se ha determinado que la normativa para conservación aporta poco a la preservación del bosque en Guanacaste (CalvoAlvarado, McLennan, Sánchez-Azofeifa, \& Garvin, 2009) y este constituye la cobertura natural principal para favorecer la conectividad en el área de estudio.

La recuperación del bosque en la región guanacasteca requiere no solo de políticas de conservación sino también de cambios en factores socioeconómicos, como por ejemplo nuevas oportunidades de empleo diferentes de las brindadas por el sector agrícola o ganadero (CalvoAlvarado et al., 2009). Será estratégico que los esfuerzos para recuperar y mantener el bosque en Guanacaste prioricen las áreas con más potencial para lograr resultados (Calvo-Alvarado et al., 2009). Considerando los hallazgos del presente estudio, dichos esfuerzos en ambas propuestas de conectividad estructural pueden enfocarse en el área más próxima a estas ya que fue allí donde se encontró mayor permanencia del bosque. Además, prioritario en las dos propuestas sería iniciar en su sector sur donde se localizaron más pérdidas de cobertura boscosa.

\section{AGRADECIMIENTOS}

Agradecemos a Heiner Acevedo Mairena, Wilfredo Segura López y Zaidett Barrientos Llosa, por sus invaluables aportes en la revisión del documento.

\section{REFERENCIAS}

Arauz-Beita, I., \& Arias-Navarro, A. (2016). Corredores Biológicos como Potenciadores del Desarrollo Local: Estudio de Caso del Corredor Biológico Alexander Skutch. Revista Universidad en Diálogo, 6(1), 67-79.

Araya-Gamboa, A., \& Salom-Pérez, R. (2015). Identificación de Sitios de Cruce de Fauna en la Ruta 415, en el "Paso del Jaguar", Costa Rica. Revista Infraestructura Vial, 17(30), 5-12.

Arias, E., Chacón, O., Herrera, B., Induni, G., Acevedo, H., Coto, M., \& Barborak, J.R. (2008). Las Redes de Conectividad como Base para la Planificación de la Conservación de la Biodiversidad: Propuesta para Costa Rica. Recursos Naturales y Ambiente, 54, 37-43. 
Artavia, A., Jiménez, M. Martínez-Salinas, A., Pomareda, E., Araya-Gamboa, D., \& Arévalo-Huezo, E. (2015). Registro de Mamíferos Silvestres en la Sección de la Ampliación de la Ruta 32, Limón, Costa Rica. Brenesia, 83-84, 37-46.

Balvanera, P. (2012). Los Servicios Ecosistémicos que Ofrecen los Bosques Tropicales. Ecosistemas 21(2), 136-147.

Broadbent, E.N., Almeyda, A.M., Dirzo, R., Durham, W.H., Driscoll, L., Gallagher, P., ... Randolph, S.G. (2012). The Effect of Land Use Change and Ecotourism on Biodiversity: A Case Study of Manuel Antonio, Costa Rica, from 1985 to 2008. Landscape Ecology, 27(5), 731-744. DOI: 10.1007/ s10980-012-9722-7

Calvo-Alvarado, J., McLennan, B., Sánchez-Azofeifa, A., \& Garvin, T. (2009). Deforestation and forest restoration in Guanacaste, Costa Rica: Putting conservation policies in context. Forest Ecology and Management, 258(6), 931940. DOI: 10.1016/j.foreco.2008.10.035

Canet-Desanti, L., Herrera, B., \& Finegan, B. (2012). Efectividad de Manejo en Corredores Biológicos: El Caso de Costa Rica. Revista Parques, 2, 1-10.

Carvajal, V., \& Díaz, F. (2016). Registro de Mamíferos Silvestres Atropellados y Hábitats Asociados en el Cantón de la Fortuna, San Carlos, Costa Rica. Biocenosis, 30(1-2), 49-58.

Centro Nacional de Información Geoambiental. (2018). Fotos aéreas Terra 1997-1998. Recuperado de http://ceniga. sinac.go.cr/fotos/

Chuvieco, E. (2010). Teledetección ambiental. La observación de la Tierra desde el espacio. Barcelona, España: Ariel.

DeFries, R., Hansen, A., Newton, A.C., \& Hansen, M.C. (2005). Increasing Isolation of Protected Areas in Tropical Forests over the Past Twenty Years. Ecological Applications, 15(1), 19-26. DOI: 10.1890/03-5258

De la Ossa-Lacayo, A. (2013). Cercas vivas y su importancia ambiental en la conservación de avifauna nativa. Revista Colombiana de Ciencias Animales, 5(1), 171-193.

Di Stéfano, J.F., Nielsen, V., Hoomans, J., \& Fournier, L.A. (1996). Regeneración de la Vegetación Arbórea en una Pequeña Reserva Forestal Urbana del Nivel Premontano Húmedo, Costa Rica. Revista de Biología Tropical, 44(2), 575-580.

Fernández, A., Recio, J., \& Ruiz, L.A. (2003). Análisis de imágenes mediante texturas: aplicación a la clasificación de unidades de vegetación. Revista Internacional de Ciencia y Tecnología de la Información Geográfica, 3, 143-159.

François, J., Reyes, J., \& Pérez, A. (2003). Evaluación de la confiabilidad temática de mapas o de imágenes clasificadas: una revisión. Investigaciones Geográficas, 51, 53-72.

García, Q., \& Abad, J. (2014). Los corredores ecológicos y su importancia ambiental: Propuestas de actuación para fomentar la permeabilidad y conectividad aplicadas al entorno del río Cardeña (Ávila y Segovia). Observatorio Medioambiental, 17, 253-298.
Gómez, O. (2014). Ancho óptimo de las rutas de conectividad biológica en el subcorredor Barbilla-Destierro, Zona Central Caribe de Costa Rica (Tesis de licenciatura). Instituto Tecnológico de Costa Rica, Cartago. Recuperado de https://repositoriotec.tec.ac.cr/handle/2238/3936

Granados-Sánchez, D., Hernández-García, M.A., \& López-Ríos, G.F. (2006). Ecología de las Zonas Ribereñas. Revista Chapingo Serie Ciencias Forestales y del Ambiente, 12(1), 55-69.

Grinand, C., Rakotomalala, F., Gond, V., Vaudry, R., Bernoux, M., \& Vieilledent, G. (2013). Estimating deforestation in tropical humid and dry forests in Madagascar from 2000 to 2010 using multi-date Landsat satellite images and the random forests classifier. Remote Sensing of Environment, 139, 68-80. DOI: 10.1016/j.rse.2013.07.008

Gurrutxaga, M., \& Lozano, P.J. (2007). Criterios para contemplar la conectividad del paisaje en la planificación territorial y sectorial. Investigaciones Geográficas, 44, 75-88. DOI: 10.14198/INGEO2007.44.04

Haddad, N.M., Brudvig, L.A., Clobert, J., Davies, K.F., Gonzalez, A., Holt, R.D. ... Townshend, J.R. (2015). Habitat fragmentation and its lasting impact on Earth's ecosystems. Science Advances, 1(2), 1-9. DOI: 10.1126/sciadv.1500052

Joppa, L.N., Loarie, S.R., \& Pimm, S.L. (2008). On the Protection of "Protected Areas". PNAS, 105(18), 6673-6678. DOI: 10.1073/pnas.0802471105

Jackson, S.D., \& Griffin, C.R. (2000). A Strategy for Mitigating Highway Impacts on Wildlife. En T. A. Messmer \& B. West (Eds.), Wildlife and Highways: Seeking Solutions to an Ecological and Socio-economic Dilemma (pp. 143-159). Massachusetts: The Wildlife Society.

LaPoint, S., Balkenhol, N., Hale, J., Sadler, J., \& van der Ree, R. (2015). Ecological connectivity research in urban areas. Functional Ecology, 29, 868-878. DOI: $10.1111 / 1365-2435.12489$

Laurance, W.F., Useche, D.C., Rendeiro, J., Kalka, M., Bradshaw, C.J.A., Sloan, S.P., \& Zamzani, F. (2012). Averting Biodiversity Collapse in Tropical Forest Protected Areas. Nature, 489, 290-294. DOI: 10.1038/nature11318

Lees, A.C., \& Peres, C.A. (2007). Conservation Value of Remnant Riparian Forest Corridors of Varying Quality for Amazonian Birds and Mammals. Conservation Biology, 22(2), 439-449. DOI: 10.1111/j.1523-1739.2007.00870.x

MacArthur, R.H., \& Wilson, I.O. (1967). Island Biography. New Jersey, United States: Princeton University Press.

Martensen, A.C., Pimentel, R.G., \& Metzger, J.P. (2008). Relative effects of fragment size and connectivity on bird community in the Atlantic Rain Forest: Implications for conservation. Biological Conservation, 141(9), 2184-2192. DOI: 10.1016/j.biocon.2008.06.008

Matteucci, S.D. (2010). La conectividad del hábitat y nuestras áreas protegidas. Fronteras, 9, 1-11. 
McRae, B.H., Hall, S.A., Beier, P., \& Theobald, T.M. (2012). Where to Restore Ecological Connectivity? Detecting Barriers and Quantifying Restoration Benefits. PLOS ONE, 7(12), 1-12. DOI: 10.1371/journal.pone.0052604

Monge-Nájera, J. (1996). Vertebrate Mortality on Tropical Highways: The Costa Rican Case. Vida Silvestre Neotropical, 5(2), 154-156.

Morera, C. \& Sandoval, L.F. (2018). Fragmentación y conectividad de la cobertura natural a nivel cantonal en Costa Rica durante los años 2000 y 2015. Revista Geográfica de América Central, 66. DOI: 10.15359/rgac.Esp-4.2

Portillo-Quintero, C.A., \& Sánchez-Azofeifa, G.A. (2010). Extent and Conservation of Tropical Dry Forests in the Americas. Biological Conservation, 143, 144-155. DOI: 10.1016/j.biocon.2009.09.020

Pérez, A.M., Sotelo, M., Ramírez, F., Ramírez, I., López, A., \& Siria, I. (2006). Conservación de la Biodiversidad en Sistemas Silvopastoriles de Matiguás y Rio Blanco (Matagalpa, Nicaragua). Ecosistemas, 15(3), 125-141.

Piquer-Rodríguez, M., Kuemmerle, T., Alcaraz-Segura, D., ZuritaMilla, R., \& Cabello, J. (2012). Future land use effects on the connectivity of protected area networks in southeastern Spain. Journal for Nature Conservation, 20(6), 326-336. DOI: 10.1016/j.jnc.2012.07.001

Quesada, M. (2016). Análisis de Vacíos de Conservación en Costa Rica: Propuesta de Reordenamiento Territorial en la Periferia de la Reserva Biológica Alberto Manuel Brenes. Revista de Geografía, 20, 75-85.

Ramos, Z.S., \& Finegan, B. (2004). Red ecológica de conectividad potencial. Estrategia para el manejo del paisaje en el corredor biológico San Juan - La Selva. Recursos Naturales y Ambiente, 49, 112-123.

Reyes, H., Morera, C., \& Reyes, O. (2017). Geografía ambiental: métodos y técnicas desde América Latina. Heredia, Costa Rica: EUNA.

Sanchez-Azofeifa, G.A., Rivard, B., Calvo, J., \& Moorthy, I. (2002). Dynamics of Tropical Deforestation Around National Parks: Remote Sensing of Forest Change on the Osa Peninsula of Costa Rica. Mountain Research and Development, 22(4), 352-358. DOI: 10.1659/0276-4741(2002)022[0352:DOTDAN]2.0.CO;2

Sánchez-Azofeifa, G.A., Daily, G.C., Pfaff, A.S.P., \& Busch, C. (2003). Integrity and Isolation of Costa Rica's National Parks and Biological Reserves: Examining the Dynamics of Land-Cover Change. Biological Conservation, 109, 123-135. DOI: 10.1016/S0006-3207(02)00145-3
Sandoval, L., Sánchez, J.E., \& Carman, E. (2013). Biología Reproductiva de la Chirrascuá (Dendrortyx leucophrys) en Costa Rica. Ornitología Neotropical, 24, 113-120.

Sanfiorenzo, A., DeClerck, F., Benjamin, T., \& Velásquez, S. (2011). Conectividad funcional para los géneros de aves Trogon, Icterus y Dendroica en el paisaje de la subcuenca del río Copán, Honduras. Agroforestería en las Américas, 48, 54-64.

Sastre, P., De Lucio, J.V., \& Martínez, C. (2002). Modelos de Conectividad del Paisaje a Distintas Escalas. Ejemplos de Aplicación en la Comunidad de Madrid. Ecosistemas, 11(2), 1-7.

Sistema Nacional de Áreas de Conservación (2007). GRUAS II: Propuesta de Ordenamiento Territorial para la Conservación de la Biodiversidad de Costa Rica. Volumen 1: Análisis de Vacíos en la Representatividad e Integridad de la Biodiversidad Terrestre. Costa Rica, Recuperado de http://www.bio-nica.info/biblioteca/ SINAC2007AnalisisVacioCR.pdf

Tapia, C.A. (2016). Análisis del cambio de cobertura forestal $2005-$ 2015 en Guanacaste, Costa Rica (Tesis de licenciatura). Instituto Tecnológico de Costa Rica, Cartago.

Torres, M.L. (2011). Funcionalidad de Estructuras Subterráneas como Pasos de Fauna en la Carretera Interamericana Norte que Cruza el Área de Conservación Guanacaste, Costa Rica (Tesis de maestría). Centro Agronómico Tropical de Investigación y Enseñanza, Turrialba, Costa Rica.

Treviño, E.J., Cavazos, C., \& Aguirre, O.A. (2001). Distribución y Estructura de los Bosques de Galería en dos Ríos del Centro Sur de Nuevo León. Madera y Bosques, 2(1), 13-25.

Valdez-Lazalde, J.R., Aguirre-Salado, C.A., \& Ángeles-Pérez, G. (2011). Análisis de los cambios en el uso del suelo en la cuenca del río Metztitlán (México) usando imágenes de satélite: 1985-2007. Revista Chapingo Serie Ciencias Forestales y del Ambiente, 17(3), 313-324. DOI: 10.5154/r. rchscfa.2010.06.041

Vargas, G. (2008). Fragmentación y conectividad de ecosistemas en el sector del proyecto geotérmico Miravalles y sus alrededores. 1975-2007. Revista Reflexiones, 87(2), 9-38.

Volk, X.K., Gattringer, J.P., Otte, A., \& Harvolk-Schöning, S. (2018). Connectivity analysis as a tool for assessing restoration success. Landscape Ecology, 33(3), 371-387. DOI: 10.1007/s10980-018-0611-6 


\section{APÉNDICE}

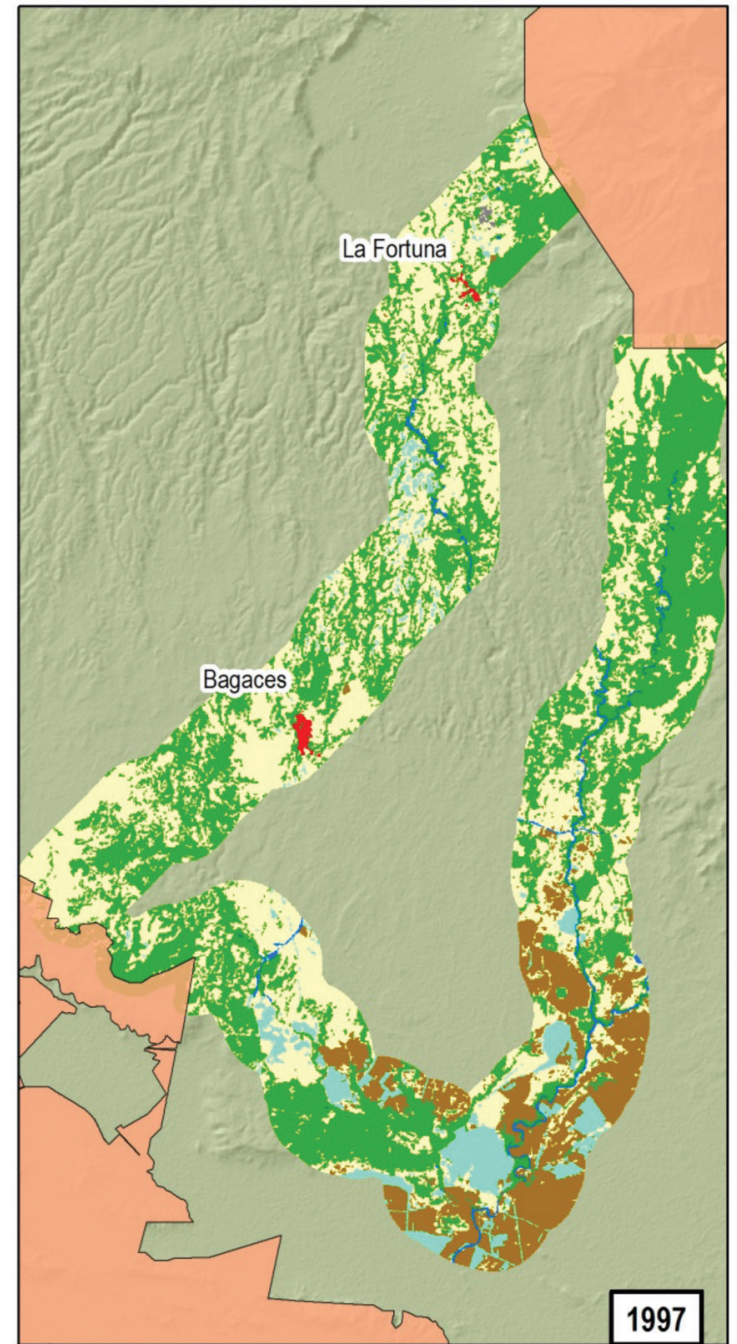

Simbología

Área Silvestre Protegida

Cobertura

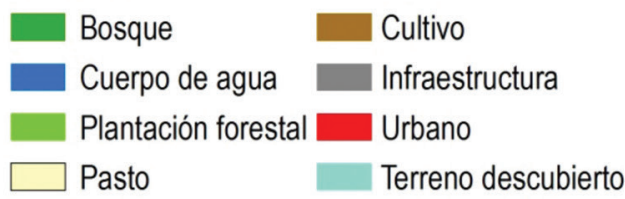

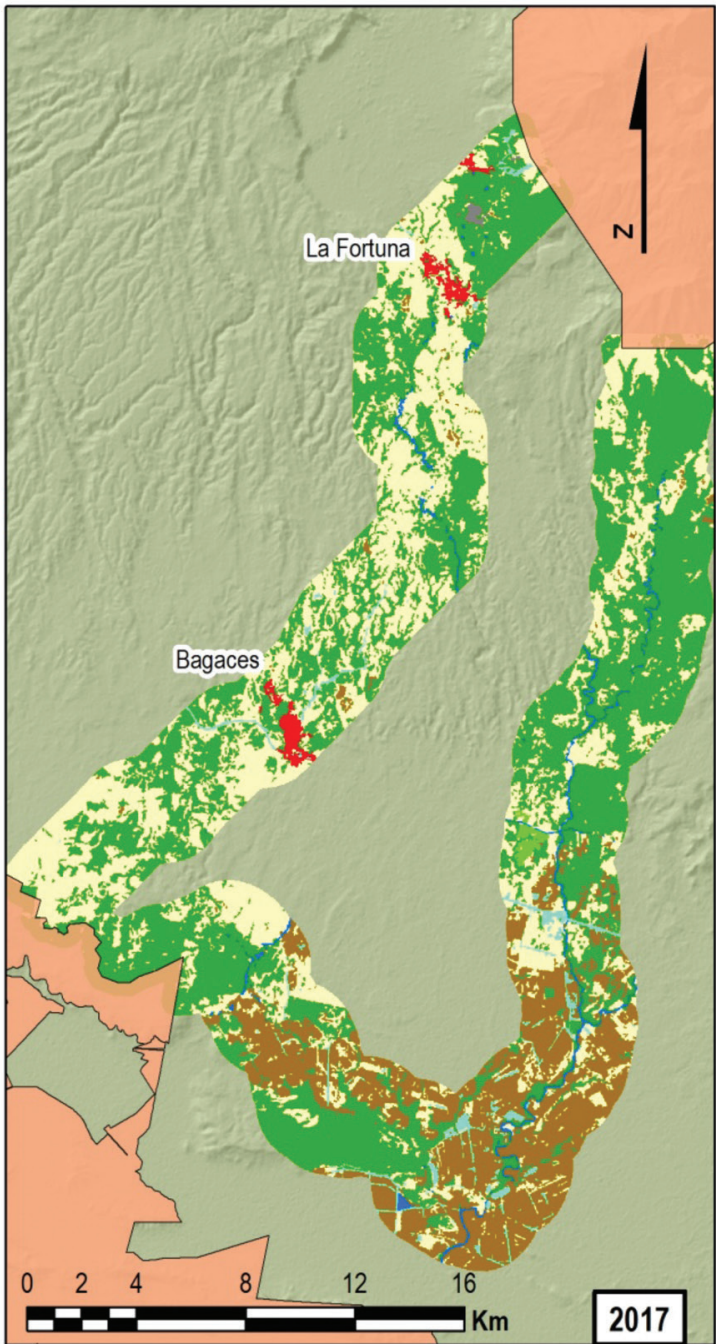

1:250.000

Proyección CRTM05

Fuente:

SNIT, IGN (2018)

Imágenes Landsat 1997, 2017

Trabajo de campo 2018

Elaborado por:

Michael A-Z

Setiembre del 2018

Fig. A. Cobertura de la tierra en las dos propuestas de conectividad estructural (al oeste la ruta oficial y al este la alterna). 


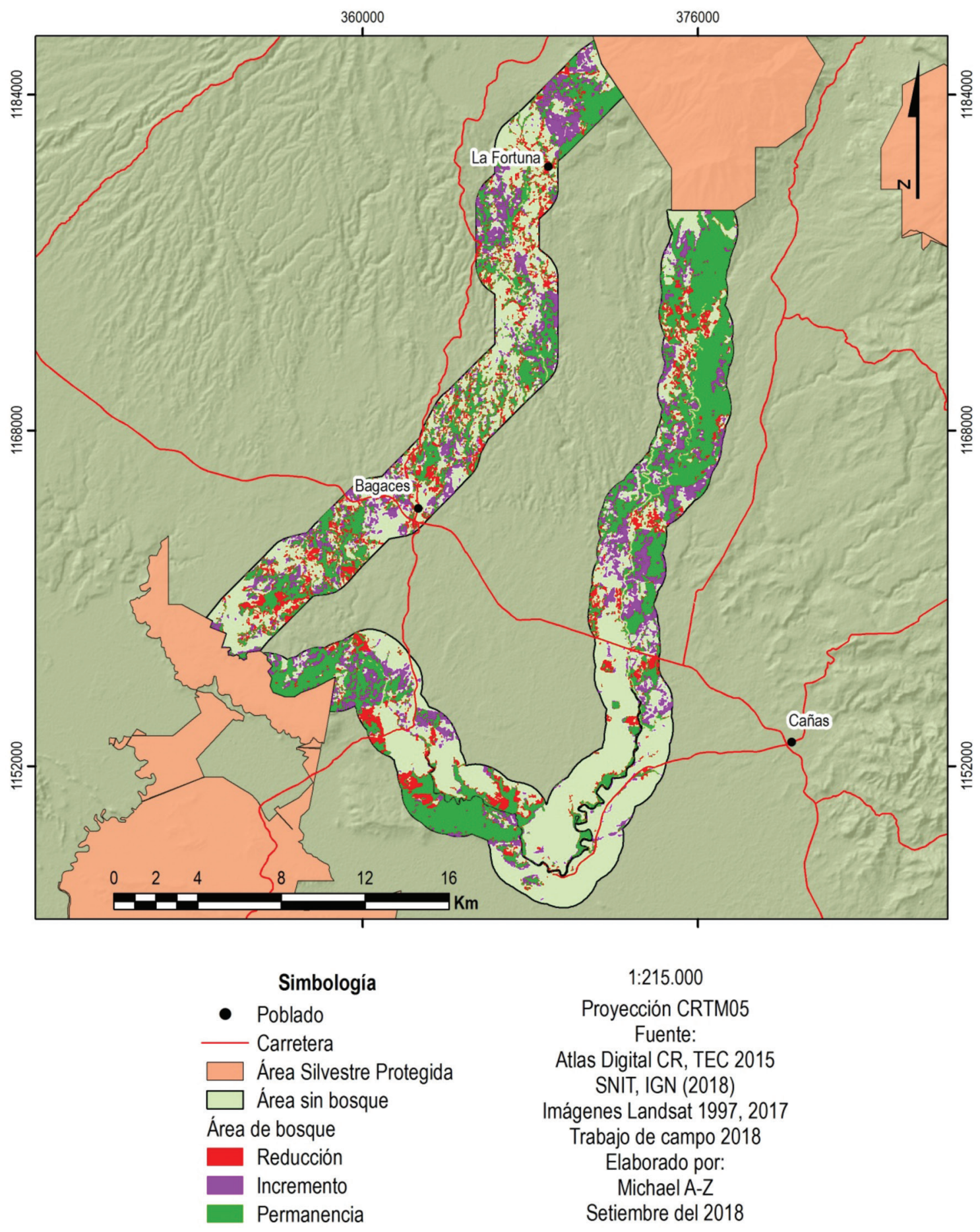

Fig. B. Reducciones e incrementos de área boscosa en las dos propuestas de conectividad. 